\title{
Téoros
}

Revue de recherche en tourisme

\section{Charlevoix et ses auberges}

\section{Normand Cazelais}

Volume 17, numéro 1, printemps 1998

Le tourisme dans un Pays à part : Charlevoix

URI : https://id.erudit.org/iderudit/1072378ar

DOI : https://doi.org/10.7202/1072378ar

Aller au sommaire du numéro

Éditeur(s)

Université du Québec à Montréal

ISSN

0712-8657 (imprimé)

1923-2705 (numérique)

Découvrir la revue

Citer cet article

Cazelais, N. (1998). Charlevoix et ses auberges. Téoros, 17(1), 51-52.

https://doi.org/10.7202/1072378ar d'utilisation que vous pouvez consulter en ligne.

https://apropos.erudit.org/fr/usagers/politique-dutilisation/ 
Normand Cazelais, géographe à Hydro-Québec et journaliste

Aar Grands Prix du Tourisme de 1995, Ferdinand Tremblay recut le Prix special du jury. Cette récompense reconnaissait au propriétaire de I'Auberge des Peupliers, de Cap-à-l'Aigle, son rôle de pionnier et d'instigateur de la renaissance des auberges tant au Québec qu'en Charlevoix. Aujourd'hui à la retraite, il a donné un second souffle à ce "produit", en lai proposant, par l'exemple de son ćtablissement et de son action, par un atvachement indefectible à ses racines régionales, un ideal d'accueil et des standards supérieurs de décoration, de confort et de restauration.

Charlevoix compte aujourd'hui plus de cinquante auberges; une quinzaine s'inscrivent dans le créneau haut-de-gamme et constituent, pour ce, une solution de rechange aux grands hôtels, en termes de service et de prestations, tout en offrant une ambiance plus intime et plus personnalisée. Dans les Cantons-de-l'Est, en Gaspésie, dans les Laurentides, dans l'Outanouais, dans toutes les régions du Québec en fait, les auberges de toute catégorie manifestent activement lcur présence et font partie du corpus touristique stratégique.

\section{MAIS POURQUOI CHARLEVOIX?}

Pays de mer et de montagne, la région possède des paysages exceptionnels. Et un cadre de vie très caractérisé. Longtemps en retrait, peu peuplé, c"est un pays compact, assez homogène où se distinguent pourtant plusieurs entités, celles des montagnes, du littoral, du plateau, de l'est et de l'ouest. L'importance du fleuve (qui est déjà une mer à sa hauteur), l"histoire des capitaines et des pilotes, des goélettes, de la navigation et du cabotage, tout comme les grandes forêts sur les hauteurs derrière, ont façonné Charlevoix. Le lien routier avec le reste du Québec n'y est apparu que tardivement, et cela se manifeste encore dans l'organisation de l'espace et du tissu social.

Il a en effet fallu attendre jusqu'au siècle dernier pour qu'un premier chemin voyer traverse Charlevoix de part en part. Le toponyme officiel de la région ne date luimême que de 1855. Avant les voies terrestres, les gens utilisaient les bateaux, goélettes et voitures d'eau, allaient d'une anse à l'autre, d'un village à l'autre par' le fleuve. Et levaient les yeux pour voir ce pays de vertige. Le train n'y changea pas grand chose: sa voie se colla à un mince lisére au bord du fleuve.

Accoudés au bastingage des blancs navires de la Canada Steamship Line, les touristes d'autrefois decouvraient sa muraille impressionmante dressée devant le SaintLaurent. Après la Deuxième guerre mondiale, avec le déclin des croisières et de la grande villégiature, la route et l'automobile ont relégué Charlevoix à la dimension d'une carte postale qu'on traversait, de préférence l'été, en se rendant ailleurs, après y avoir laissé quelques soupirs d'admiration... Ses visiteurs y séjournatent en moyenne moins d'une journé: beaucoup y passaient mais Charlevoix en tirait peu de retombés économiques. Elle en éprouvait plutôt certains inconvénients. comme des attentes aux traversiers, des engorgements de circulation, des flambées de prix.

Remarquable par sa diversité, son nombre et son rayonnement, le réseau des auberges a changé la donne en contribuant à refaire de Charlevoix unc destination. une région touristique de séjour et recherchée comme telle. Même si l'élan initial s'est ralenti et si le stock des etablissements s'est stabilisé, le renouveau et l'éclosion des auberges ont permis d'y allonger les sejours. Et de constater qu'au-delà des beaux paysages il y avait une vie, une culture, un patrimoine, des gens. Et pas seulement en éte, mais en toutes saisons.

Ce cas illustre bien l'identification pouvant s'établir entre une région et ses auberges qui se sont affirmés comme un label à la fois collectif et régional, en raison de cette cohésion interne et de leur évidente relation avec lá vie touristique et culturelle de Charlevoix.

Trois éléments valent d'être étudiês de plus près, pour ce qu'ils révèlent de la nature profionde des auberges qui se démarquent des autres lieux d'hébergement, tant dans Charlevoix qu'ailleurs au Québec et même en Amérique du Nord, bien davantage par' une conception de l'accueil qu'ils représentent et véhiculent que par des normes de construction ou de service (qui, elles, ventilent les catégories d'établissements). 


\section{LA LOCALISATION}

Conrad Hilton, à qui on peut tout de mème reconnaître une certaine compétence dans le domaine de l'hôtellerie, quelles qu'en soient les manifestations, aimait répéter qu'il y a trois conditions à satisfaire pour assurer le succes d'un établissement: le site, le site et encore le site.

Apparus avec l'expansion de l'automobile, les motels, comme leur nom l'indique (motor hotels), fréquentent les abords des routes. Leur accès facile, immédiat, jouxté à une possiblité de réduire le service (et, en conséquence, les tarifs) au strict minimum, a fait leur fortune et explique encore aujourd' hui leur popularité, même si les goûts et attentes de la clientèle ont changé et entraîné une forme de raffinement de leur décor et de leurs prestations.

On ne compte plus, dans les répertoires, les Hôtel de la Gare, Que l'on change la gare de chemin de chemin de fer pour un terminus d'autocar's, une place du marché, un aéroport, un carrefour d'autoroutes, le voisinage de grands carrefours de commerce ou d'affaires, et l'on saisit les lois qui ont régi et régiront encore longtemps la localisation de la plupart des établissements hôteliers. Y échappent les hôtels dits de villégiature, également connus sous l'appellation de resorts, qui recherchent des lieux retirés, au milieu de paysages attrayants, à portée d'une nature invitant à la détente ou à l'activité sportive.

La localisation des auberges obéit à des facteurs similaires à ceux prévalant pour ces derniers établissements. Elles ouvrent le plus souvent leurs portes en des lieux retirés, calmes, offrant à la vue des spectacles naturels remarquables. Cela qualifie déjà Charlevoix dans son ensemble qui, très tồt, est devenu un havre d'artistes, un espace de ressourcement et d'inspiration. Plus encore, au sein même de cette région si richement dotée, les auberges $s$ tepanouissent dans les meilleurs endroits si l'on peut dire, pour l'essentiel à flane de montagne ou devant le fleuve.

Une rapide analyse de la répartition des auberges de Charlevoix, le terme étant entendu en son sens le plus large, donne ce qui suit: $24 \%$ se situent dans la partie ouest (avec une nette concentration dans les environs immédiats de Baie-SaintPaul), $42 \%$ dans la partie centrale, le long 362 qui longe le fleuve en suivant l'abrupt du massif montagneux (avee une antenne a l'ile aux Coudres), et $34 \%$ dans la partic est (essentiellement à Pointe-au-Pic, La Malbaie et Cap-à-l'Aigle).

\section{L'ARCHITECTURE}

Dès le départ, les auberges ont privilégié les bâtiments ayant du caractère, évoquant l'histoire et surtout l'authenticité du terroir, parfois aux dépens du confort, de la fonctionnalité... et de la rentabilité. Il suffit d'en discuter avec les aubergistes pour apprendre, avec moults détails, à quel point ils ont trop souvent dû composer avec des maisons qui, pour être belles et charmantes, ont demandé des trésors d'imagination et de débrouillardise à la fois pour préserver leur cachet, satisfaire les besoins de la clientèle et les exigences des diverses offines gouvernementales (sorties d'urgence, mesures contre le feu, sanitaires, etc.).

Ce fut et c'est leur marque de commerce. Peut-on imaginer une auberge, dans Charlevoix de surcroît, dans un bâtiment carré, anonyme, fonctionnel peut-être mais ne dégageant aucune convivialité? Poser la question, c'est y répondre. Toute auberge ne correspondant pas dans son enveloppe extêrieure à l'image de Charlevoix était et serạ vouée à l"échec. L'architecture est indissociable du produit.

\section{LE NOM}

Il s'agit sans doute de l'élément le plus révélateur. Les auberges ne peuvent avoir un nom quelconque. La puissance des toponymes: des destinations attisent les départs par la force leurs noms. Rio de Janeiro, Hong Kong, Bali, Venise en sont des exemples. Pour une auberge, avoir un nom qui interpelle, qui attire l'attention sinon déjà le goût d'en savoir plus, de vérifier sur place, c'est déjà une gros morceau.

En Charlevoix, les hôtels et motels peuvent se contenter d"avoir des noms passepartout: Belvédère, Plaza, Bellevue, Leclerc, Plaza, Point de vue et j'en passe. Cela est impensable dans le cas des auberges qui s'appellent Des Peupliers, La Pinsonnière, La Muse, La Maison Otis, Des Trois Canards, La Source, Sur la Côte, La Coudrière. Et ce qui est vrai pour Charlevoix l'est partout ailleurs. Une auberge ne peut se permettre la banalité.
J'essaic d'imaginer à quoi pouvaient penser Cartier et ses matelots quand ils naviguèrent, pour une première fois, au pied Charlevoix. Ils avaient traversé l'Atlantique sur leurs coquilles de noix, planté une croix à Gaspé, pris possession de ces terres au nom de leur souverain. Rencontré des Indiens. Ils avaient connu les vagues et les brumes du golfe, la cote tourmentée de la Terre de Caîn au nord de I'Anticoste. Que pouvaient-ils penser devant cette muraille de roes et de forêts degringolant dans la mer?

Peut-être rien, en fait. Pour eux, après avoir vu les hautes parois du fjord qui $s^{+}$enfonçait plus au nord vers des aires inconnues, ce littoral n'etait-il qu'une masse hirsute et écheveléc contre laquelle glissaient des vents dangereux. Peut-être leurs yeux furent-ils plus naturellement portés vers la côte du Sud, plus amène, frangée d'îles, ouverte sur l'horizon et de lointaines montagnes bleutées tout au fond.

Ce n'est qu"à la fin du XVII" siècle et plus tard que le paysage changea, qu'apparurent champs et hameaux, d'abord à l'entréc des vallées puis sur les hauteurs des plateaux. Maisons, églises, quais, clôtures et bosquets, chemins et terres labourées, l'espace s"humanisa, devint celui que nous connaissons.

Et encore. Il fallut attendre longtemps, jusqu'au siècle demier pour que la route traverse Charlevoix de part en part. Le toponyme officiel ne date lui-même que de 1855. Avant les voies terrestres, les gens utilisaient les voitures d'eau, allaient d'un village à l'autre par le fleuve. Et levaient les yeux pour voir ce pays de vertige. Le train n’y changea pas grand chose: sa voie se colla à un mince lisêré au bord du fleuve. Il fallait toujours jeter la tête en arrière et regarder vers le haut.

Nos regards d'aujourd' hui sont différents. Au cap Tourmente, la route attaque la montagne, grimpe hardiment. A PetiteRiviêre-Saint-François, Baie-Saint-Paul, Saint-Josepth-de-Ia-Rive, Saint-Irénée. quel que soit l'endroit, nous arrivons des hauteurs: Charlevoix s'étale à notre vue devenue panoramique.

Faut-il s'étonner qu'il soit devenu un havre d'artistes?

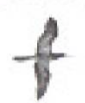

